\title{
Design and Implementation of a Stand-Alone Remote Terminal Unit
}

\author{
Prachi Sharma ${ }^{1}$, Mr. Mandeep Singh ${ }^{2}$ \\ ${ }^{I}$ (M.E Student, Electrical \& Instrumentation Deptt., Thapar University, Patiala, India) \\ ${ }^{2}$ (Assistant Professor, Electrical \& Instrumentation Deptt., Thapar University, Patiala, India)
}

\begin{abstract}
A Remote Terminal Unit (RTU) plays a vital role in substation automation. It consists of various sections that can handle different types of field parameters. This paper discusses a stand-alone RTU on a single board and its various sections: analog inputs section, digital inputs section and digital outputs section. The main objective of this paper is to check the accuracy of measurement of field inputs by the controller. A number of testing is done on each type of analog input to prove its accuracy.
\end{abstract}

Keywords: Analog Inputs, Current Transformer, Digital Inputs, Digital Outputs, Potential Transformer, Remote Terminal Unit, Substation automation.

\section{Introduction}

In the present scenario, the world is moving towards automated systems. RTUs, in a system, gather information and are responsible for sending/receiving and executing the commands issued from the control centre. A remote terminal unit is a microprocessor-controlled electronic device that interfaces objects in the physical world to a central unit or SCADA by transmitting the required data to the system and sometimes by using messages from the central unit to control connected objects [1].

An RTU monitors the field digital and analog parameters and transmits data to the Central Monitoring Station. It contains setup software to connect data input streams to data output streams, define communication protocols, and troubleshoot installation problems.

An RTU may consist of one complex circuit card consisting of various sections needed to do a custom fitted function or may consist of many circuit cards including CPU or processing with communications interface(s), and one or more of the following: (AI) analog input, (DI) digital input, (DO/CO) digital or control (relay) output, or (AO) analog output card(s) [2]. It consists mainly from three major parts (sensors, microprocessor or controller, Communications parts). Each RTU is composed from the sensors that provide the required data for a certain application, the microcontroller which is the most important part of the RTU that collect the data from the sensors, process it and give it to the communication part for delivering it to the central unit [2].

\section{Literature Review}

RTUs were once "dumb" devices that served as an extension of the central computer to the local Input/output. They had no intelligence but could only read the inputs and activate the control outputs. With the development and use of 8 -bit, 16-bit and 32-bit microcontrollers, RTUs are now dedicated microcomputers with stand-alone capability [3]. With the RTUs sharing data base management with the central computer, the central computer becomes an operator interface device.

Chen Qi.et.al developed a low-power remote terminal unit (RTU) based on GPRS/GSM (general packet radio service / global system for mobile communication) hybrid communications for data acquisition and transmission from distributed monitoring points in industrial environments. During the GPRS network congestion problem, the Short Message Service (SMS) mode is used for data transmission with guaranteed integrity. The ultra-low powered MSP430 microprocessor is used in this RTU. Thus, power consumption obtained during the working cycle was 200 milliWatts $(\mathrm{mW})$ and $0.2 \mathrm{~mW}$ during the low power mode. Also, no data losses were obtained with the hybrid communication system during a $48 \mathrm{~h}$ test [4].

Chen Peijiang and Jiang Xuehua designed and implemented a remote monitoring system based on GSM. The system includes two parts: monitoring centre and the remote monitoring station. The communication between these two parts is done using a TC35 communication module of GSM. MSP430F149 MCU is used in the monitoring station. The result of demonstration showed that the system can monitor and control the remote communication between the monitoring centre and the remote monitoring station [5].

Chang.et.al developed a DNP3.0 protocol based on the high performance remote terminal unit (RTU) which was applied to the feeder automation system of substations. The Harvard architecture advanced microcontroller using pipeline processing technology would be used as the control core, to improve the system efficiency. The RTU proposed in this paper could measure the voltage and current of the feeder, monitor the 
feeder line branch switch status, digital relay, and utilize DNP3.0 and feeder automation host machine [6].

Aamir.et.al has done a comparative study of two different selections of CPU for designing an RTU based on performance measurement in view of energy management applications. The two CPUs are Programmable Logic Controller (PLC) and Field Programmable Gate Array (FPGA). The study showed that FPGA based RTU possesses unique features like encryption support, radio support and large memory area while PLC based RTU exhibits limited features. An optimized RTU was proposed based on the significant parameters that facilitate the optimized design [7].

\subsection{Digital Inputs (DI) Section}

\section{Description of RTU Sections}

This section is made to provide feedback to the controller about the status of various field devices. These digital inputs can be the status of circuit breaker, isolator and sequence of events. Hence, this section takes inputs from field relays that are connected to various devices. These inputs are either "1" or " 0 ". Thus, these are called digital inputs. In this project, there are 12 digital inputs with 1 common terminal. This section works upon $+24 \mathrm{~V}$ and can also be called as Feedback Section.

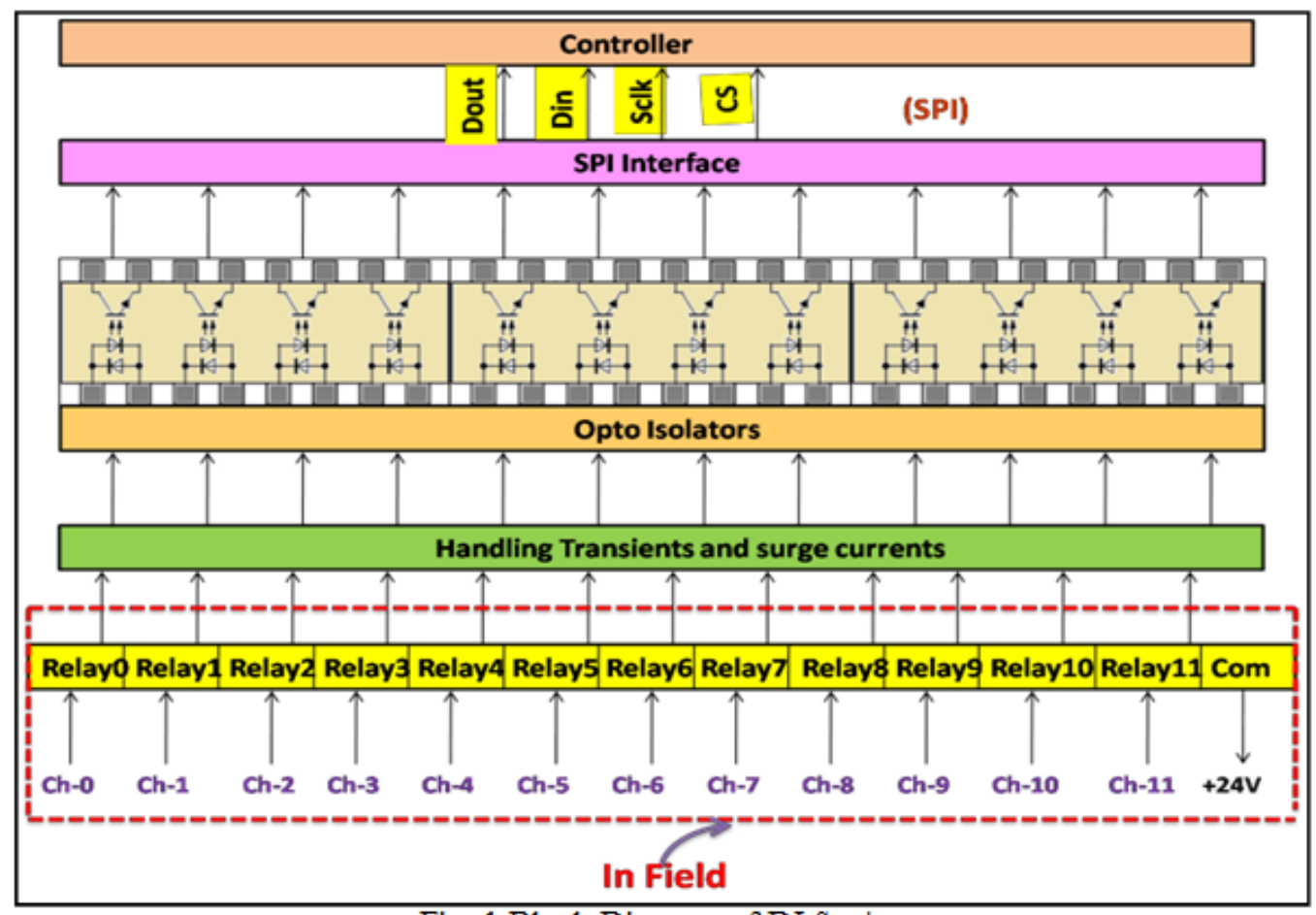

Fig. 1 Block Diagram of DI Section

As can be seen in Figure 1, there are a number of relays connected to various devices in the field. The outputs of these relays are given as digital inputs to the DI section. These inputs are passed through some protecting devices like MOV, TVS etc. for handling transients and surge currents. It is required to isolate controller from field signals, so optoisolators are necessary to provide such isolation. As shown, now, outputs of isolators are sent to the controller using Serial Peripheral Interface.

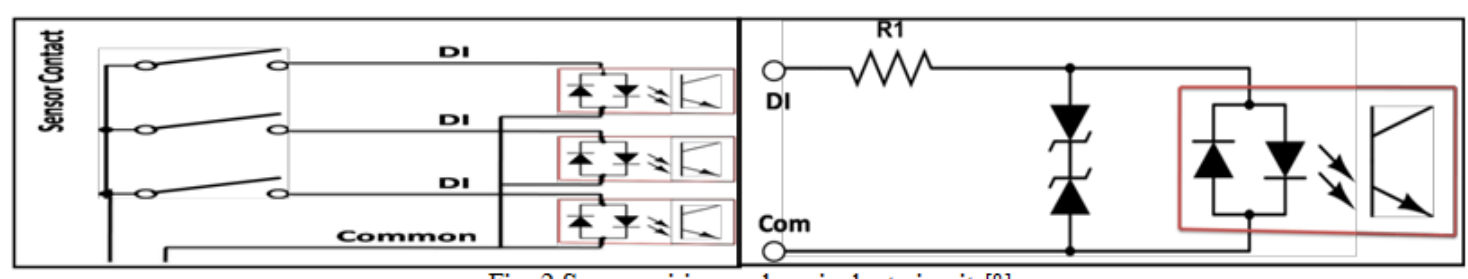

Fig. 2 Sensor wiring and equivalent circuit [8]

The connection of field relays to sensors and to the DI section is shown in Figure 2. Relays are operated according to the status of the sensors and are then given to the controller by passing them from optoisolators. The figure 2 also shows the equivalent circuit of DI section for only one channel of digital inputs. It shows the protection device (MOV or TVS diodes) and necessary passive components to be used in its circuitry. 


\subsection{Digital Outputs (DO) Section}

As DI section was for monitoring, the purpose of DO section is for controlling the field devices like circuit breaker etc. After continuous monitoring of field devices by DI section, if there is any need arises to control any of the field devices a $t$ a particular time, the controller will give command to the corresponding relays in the DO section and then these relays will send commands, in the form of digital outputs, to the field devices connected to them and so, in this way, field devices are controlled. In this section, 8 digital output channels are used. The relays used have 3 terminals: Common, NO (Normal Open), NC (Normal Closed). Hence, the relays are SPDT (Single Pole Double Throw).

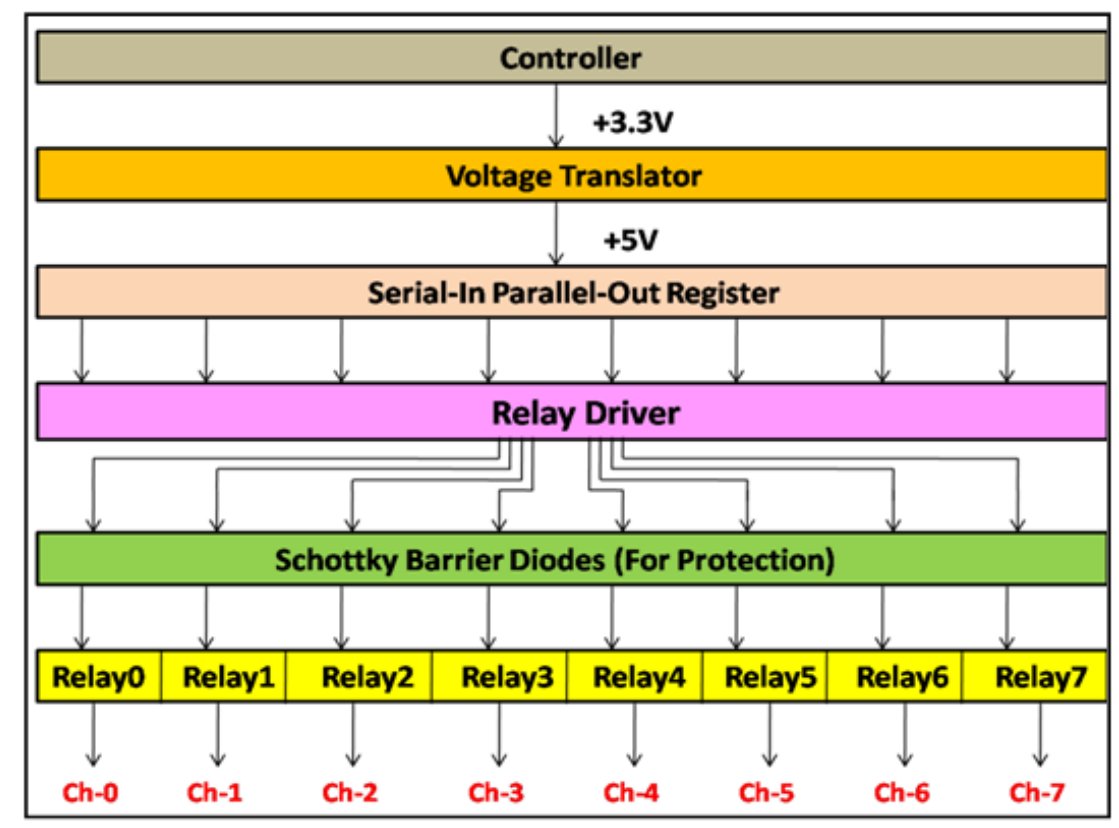

Fig. 3 Digital Outputs Section

As shown in Figure 3, after taking feedback from the DI section, controller wants to control the field devices directly by on-board relays. For this purpose, a Serial-In-Parallel-Out (SIPO) register is required to convert serial data from the controller into the parallel one for operating the relays connected in parallel. The Controller and SIPO register works on $+3.3 \mathrm{~V}$ and $+5 \mathrm{~V}$ respectively. Thus, a voltage translator is needed to convert these voltage levels. Now, as shown in this figure, a relay driver is always required for driving the relays which will then control the field devices connected to them. In this way, control action takes place.

\subsection{Analog Inputs (AI) Section}

This section is the most complex and critical one. It gives information about on site physical parameters, line voltages, line currents to the controller continuously. This section consists of various inputs according to the application:
1. Auxiliary Inputs.
2. Voltage Inputs.
3. Current Inputs.
4. DC Inputs. 


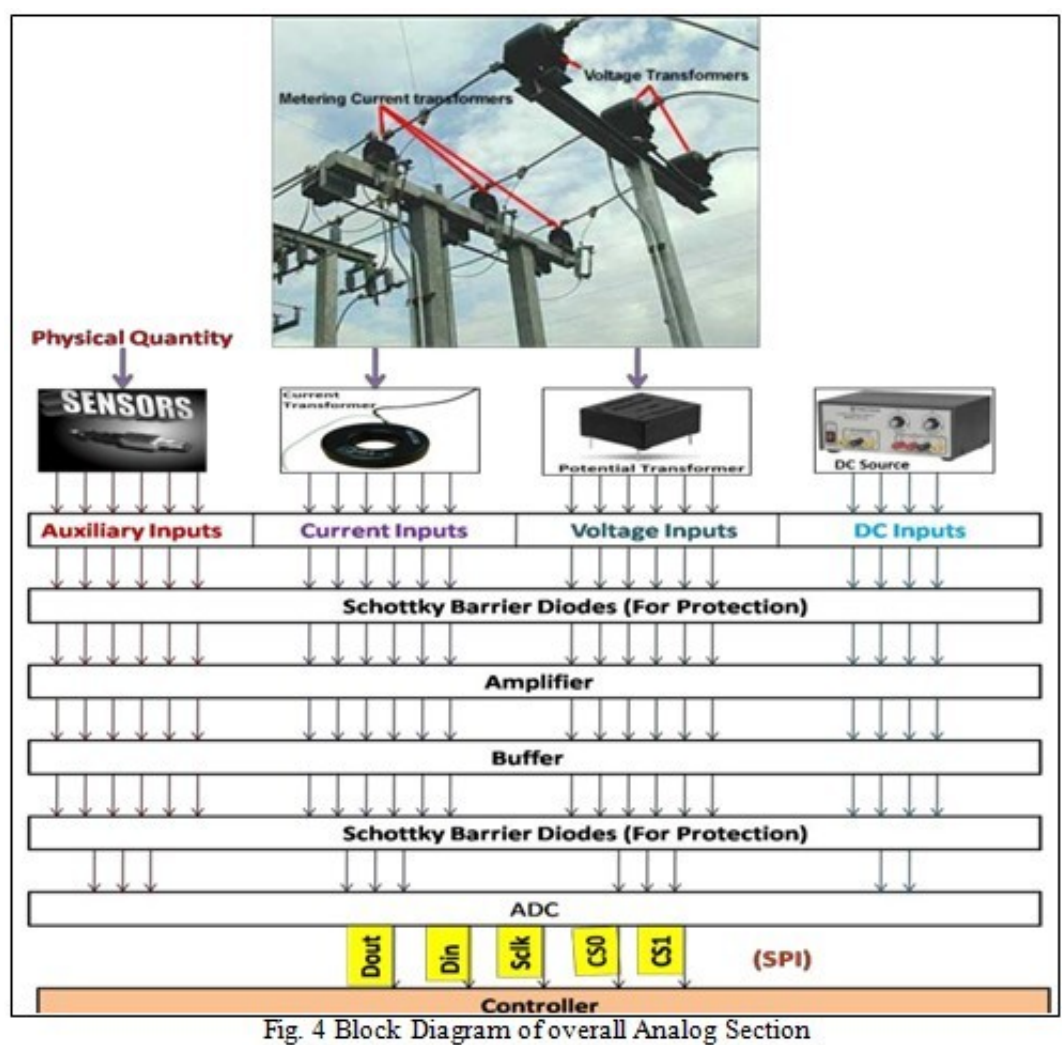

In Figure 4, processing of all four types of inputs is shown. According to the figure, processing of each type of input is explained one-by-one.

\subsubsection{Auxiliary Inputs}

In the field, physical parameters like pressure, temperature etc. is sensed by various sensors. These sensors provide their output in the range of 4-20 mA. After passing through resistor network, these current signals are converted into voltage signals. Here, 3 auxiliary input channels are used. From Figure 4, these signals are given to schottky barrier diodes for protection against transients. Now, these signals are given to amplifiers which act as buffer and provide noise free signal. These analog signals are then digitized with the help of Analog-to-Digital Converter (ADC). These digitized signals are given to the controller with the help of serial peripheral interface as shown in the figure.

\subsubsection{DC (Direct Current) Inputs}

In this section, two DC input channels are used: DC1 and DC2. DC inputs are coming from DC source as shown in Figure 4. Here, DC inputs range from $80 \mathrm{~V}$ to $290 \mathrm{~V}$. These signals undergo the same procedure as the auxiliary inputs do. In this way, these DC inputs are continuously measured and monitored.

\subsubsection{Voltage and Current Inputs}

Voltages and currents in the transmission lines are of very high value. These high values can't be measured directly. They have to be converted into some lower values which can be conveniently connected to measuring and recording instruments. For this purpose, current transformers (CT) and potential transformers (PT) are used for line currents and line voltages respectively.

Potential transformers (PT) (also called voltage transformers (VT) are a parallel connected type of instrument transformer. They are designed to present negligible load to the supply being measured and have an accurate voltage ratio and phase relationship to enable accurate secondary connected metering. The PT is typically described by its voltage ratio from primary to secondary (E.g. 600:120). Burden and accuracy are usually stated as a combined parameter due to being dependent on each other. In current transformer, the primary winding is the main conductor passing through the centre of the core. The secondary winding is uniformly distributed around the toroidal core. Essentially, all the flux which links the primary conductor also links the secondary winding.

The rating of current transformers used on transmission lines is approx. $800 / 1200 \mathrm{~A}$ to $1 \mathrm{~A}$ or $5 \mathrm{~A}$ and rating of potential transformers is approx. $220 \mathrm{KV}$ to $110 \mathrm{KV}$. The output of these transformers is then given to 
another current and voltage transformers of lower rating as shown in Figure 4. The ratings of these current and voltage transformers are $1 \mathrm{~A} / 5 \mathrm{~A}$ to $\sim 200 \mathrm{mV}$ and $110 \mathrm{KV}$ to $\sim 200 \mathrm{mV}$. According to the figure, the output of PT is amplified and then converted into digital form with the help of ADC. Finally, these signals are given to the controller.

\subsection{Test 1: Testing of Auxiliary Inputs of Analog Section}

\section{Testing Methodology and Results}

There are 3 auxiliary input channels. Figure 5 shows whole processing of these auxiliary input channels. This test is performed to know how accurately the status of field sensors is measured and detected by the controller using ADC.

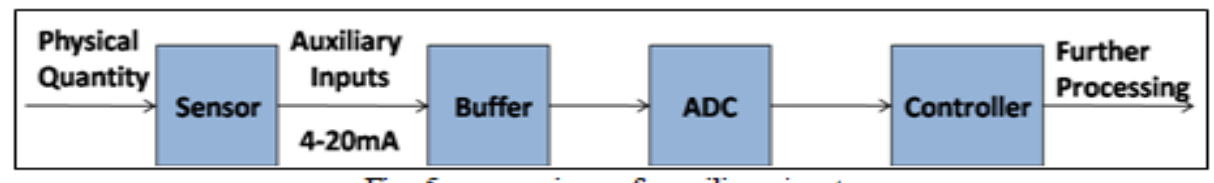

Fig. 5 processing of auxiliary inputs

Here, the physical quantity can be temperature, pressure etc. These physical quantities are sensed by field sensors which gives their output in the range of 4 to $20 \mathrm{~mA}$. These signals, after converting them to voltage signals, are given to a buffer to get a noise free signal. These signals are then applied as inputs to ADC which sample and digitize these signals as the controller accept only digital signals. For monitoring the analog parameters, there are various sensors in the field.

\subsubsection{Channel 1 Measurements}

The steps for this testing of input channel 1 are concluded as:

Step 1: A variable resistor is used, which works on $+24 \mathrm{~V}$ and has various resistances to provide current in the range of 4 to $20 \mathrm{~mA}$. The input channel 1 of AI card is tested by connecting it to the variable resistor and set its resistance accordingly to provide $4 \mathrm{~mA}$.

Step2: The 4mA current is passed to the resistor network in the AI card which converts it into voltage (mV).

Step3: To make the signal noise free, this voltage is then passed through a buffer.

Step4: The output of the buffer is an analog signal. This analog signal is given as an input to the ADC.

Step5: The ADC count corresponding to $4 \mathrm{~mA}$ is calculated according to the formula given by equation (1), given in the datasheet as:

$A D C$ Count $=(2 N \times A I N \times G A I N) / V R E F \quad$ (1)

Step6: After applying voltage to the ADC, the analog signal is converted into the digital one by taking samples and then, ADC calculates the voltage from these samples. The applied formula is given in (2):

Calculated Voltage $=($ ADC count $\times 2.5) /(224 \times 2)(2)$

Step7: Applied input is $4 \mathrm{~mA}$ and voltage is also calculated with the help of samples, so now resistance can be calculated with the help of ohm's law.

Step8: Now, according to this calculated resistance, current is calculated. It should be equal to the input current which tells the accuracy of the AI card.

Step9: In the end, error percentage between input current and calculated current is determined by using the following formula as:

Error $\%=(($ Calculated current - Input current $) /$ Calculated current $) \times 100$

Step 10: All the above steps are followed for $8 \mathrm{~mA}, 12 \mathrm{~mA}, 16 \mathrm{~mA}$ and $20 \mathrm{~mA}$ for the same input channel.

Step11: A graph is plotted between Input current and calculated output current as shown in Figure 6 as shown below.

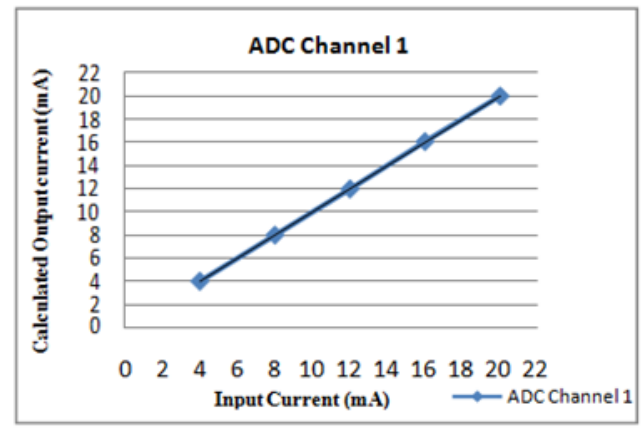

Fig.6 Graph between I/p \& Calculated current for channel 1 
This figure shows a linear graph between input current and calculated output current. It means the output current follows the input current accurately which is the desired result.

\subsubsection{Channel 2 Measurements}

The same steps are followed as for channel 1 and so a graph is plotted between input and output as shown in Figure 7.

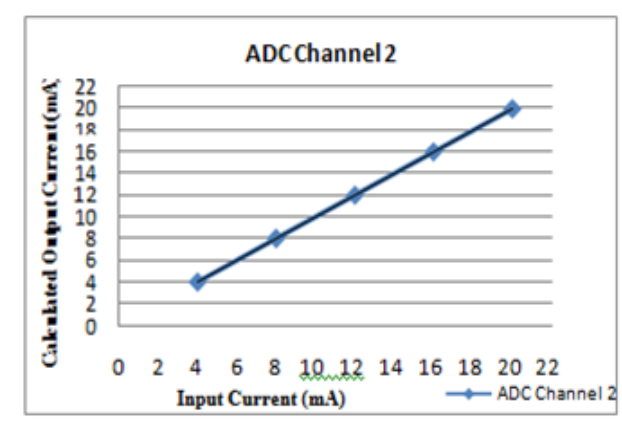

Fig. 7 Graph between I/p \& calculated current for channel 2

This figure shows a linear graph between input current and calculated output current. It means the output current follows the input current accurately which is the desired result.

\subsubsection{Channel 3 Measurements}

The same steps are followed for this channel 3 as followed for channel 1 and 2. The graph obtained for this input channel is shown in Figure 8 below.

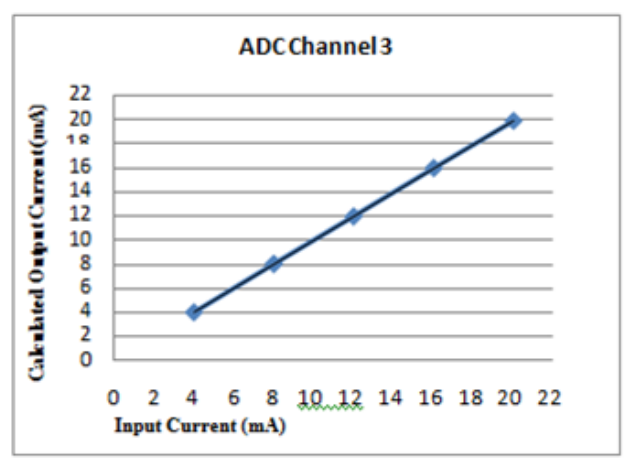

Fig. 8 Graph between $\mathrm{I} / \mathrm{p}$ \& calculated current for channel

This figure shows a linear graph between input current and calculated output current. It means that the output follows the input accurately which is the desired result.

\subsection{Test2: Testing of current inputs with the help of current transformer}

This testing is done to know at which value of input current, CT gives accurate output voltage ( $200 \mathrm{mV}$ ) and at which input, the output voltage of CT starts saturating. There are 3 current input channels in AI section. Figure 9 explains the whole processing of current inputs.

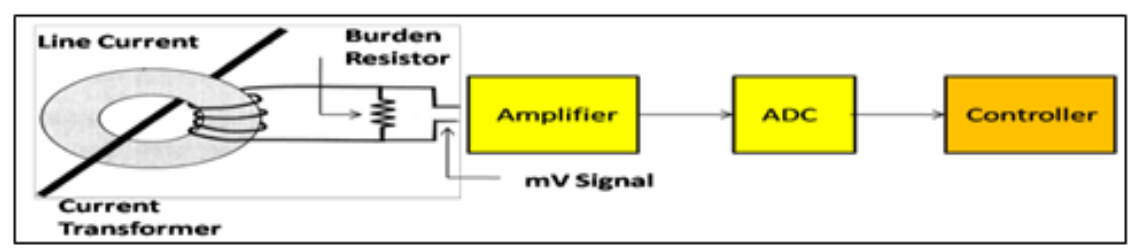

Fig. 9 Block Diagram representing processing of current inputs

This figure shows that the $1 \mathrm{~A} / 5 \mathrm{~A}$ current coming from the high rating current transformers, installed in series with the transmission line, are converted into some lower valued signal. The value of this signal depends upon the burden resistor. Now, the obtained mill volt $(\mathrm{mV})$ signal is passed through a unity gain amplifier or a buffer to get a noise free signal. This analog signal is then digitized using ADC and the output of that ADC is sent to the controller. Figure 10 shows the circuit diagram for the current transformer. 
In this testing, current of $1 \mathrm{~A}$ and $5 \mathrm{~A}$ is given to the $\mathrm{CT}$ inputs with the help of Omicron. Omicron is a device which provides current, voltage and frequency at different ranges.

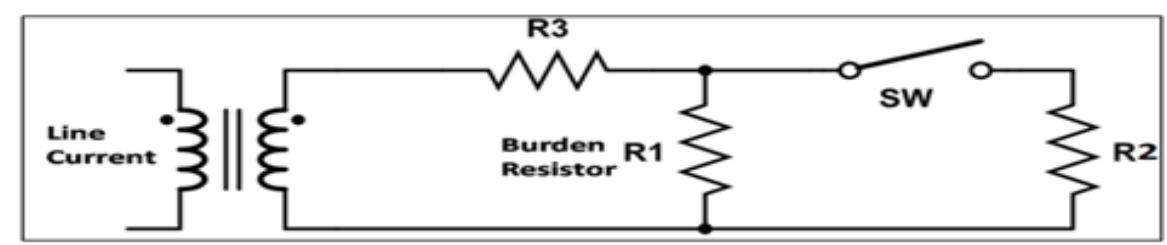

Fig. 10 Circuit Diagram for Current Transformer

As shown in Figure 10, the output of current transformer depends upon burden resistor (R1). If input is $1 \mathrm{~A}$, then only R1 has to be set. If we want to measure $5 \mathrm{~A}$ current, then the switch (SW) should be closed and resistor R2 has to be set. These resistors are set to get the required output of $200 \mathrm{mV}$.

\subsubsection{Measurement of $1 A$ current}

The steps for this testing are concluded as follows:

Step 1: At the input of CT, the current starting from 0.2A is applied with the help of Omicron.

Step2: The output voltage, across a burden resistor, corresponding to the applied input current is measured.

Step3: The expected voltage across the burden resistor is also calculated for each value of input current.

Step4: Here, these calculations are continued up to the input current of 4.5A.

Step5: The accurate output voltage of CT is obtained at 1A input current and the output waveform of CT starts saturating at the input current of $3 \mathrm{~A}$ when seen through the oscilloscope.

Step6: After following all the above steps, a graph is plotted between input current and measured voltage at burden resistor as shown in Figure 11.

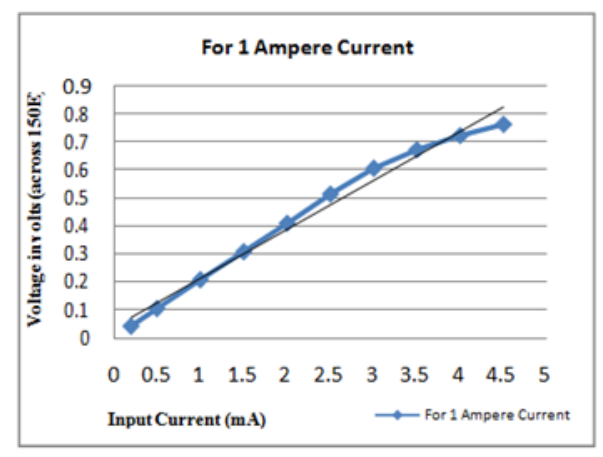

Fig. 11 Graph between I/p \& Measured Voltage for 1A Current

This figure shows that the graph between input current and the measured voltage is linear up to an input current of $3 \mathrm{~A}$, after that is starts saturating. Thus, the $\mathrm{CT}$ accurately converts the coming field input into a value that can be measured by the controller.

\subsubsection{Measurement of 5 A current}

The range of input current taken is from $5 \mathrm{~A}$ to $10 \mathrm{~A}$. The only difference, in this case, is that the output is calculated across the parallel combination of R1 and R2 rather than only R1. This testing follows the same steps as those for $1 \mathrm{~A}$ input current. A graph is plotted between input current and the output voltage as shown in Figure 12.

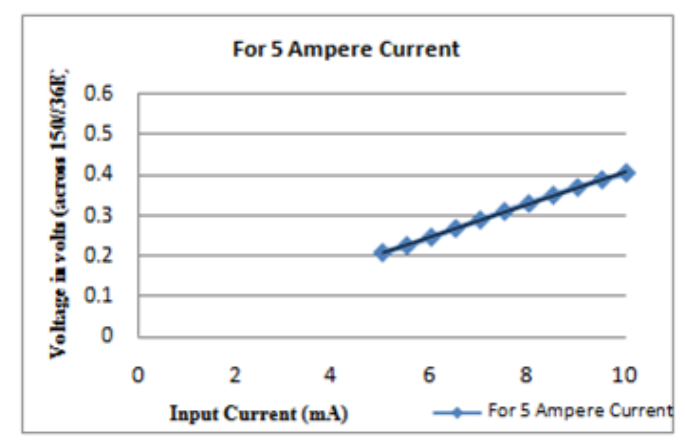

Fig. 12 Graph between I/p \& Measured Voltage for 5 A Current 
This figure shows that, in this case, the graph between input current and the measured voltage is a straight line which is the desired result. Here, the starting input current is high (i.e. 5A) and the output is in $\mathrm{mV}$, so it can not be a linear graph as was expected. But, here also, the CT accurately converts the coming field input into a value that can be measured by the controller.

\subsection{Test 3: Testing of Voltage Inputs using Potential Transformer (PT)}

This testing is done to check at which particular input voltage, output voltage at the secondary side is accurate and from which input voltage, output voltage of PT starts to saturate. There are 3 voltage channels in this project. Figure 13 shows the processing of voltage inputs.

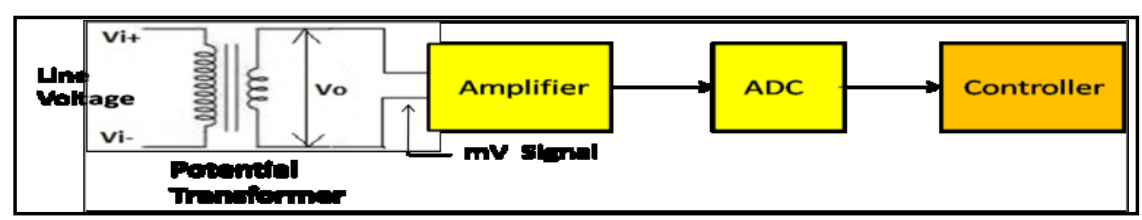

Fig. 13 Block Diagram representing processing of voltage inputs

In this figure, the PT converts high voltage coming from the high rating potential transformer installed in parallel with the transmission lines, into a low-valued signal of some $\mathrm{mV}$ range. This signal is then given to a unity gain amplifier or a buffer which results in a noise free signal. The ADC then digitizes the incoming analog signal and sends it to the controller. The steps regarding this test can be concluded as follows:

Step 1: At the primary side of PT, a number of voltages are applied starting from $0 \mathrm{~V}$ until that input voltage at which the output waveform starts saturating.

Step2: $A$ fter $128.05 \mathrm{~V}$ input current, the output voltage starts saturating and at $110.3 \mathrm{~V}$ input, accurate result is obtained.

Step3: A graph is plotted between primary and secondary voltage of the PT as shown in Figure 14 as shown below.

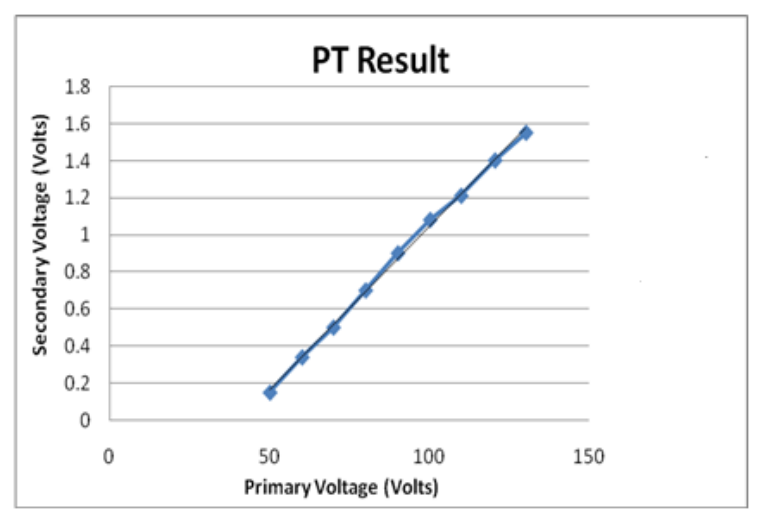

Fig. 14 Graph between Vsecondary \& Vprimary of PT

This figure shows that the graph between primary and secondary voltages of a PT is linear. It means the secondary output voltage is following the primary input voltage which is the desired result. Thus, PT accurately converts the coming input into that value that can be measured by the controller.

\subsection{Testing of DC1 and DC2}

DC measurement is very important as it has to continuously measure that what dc is being received from the dc source and what $\mathrm{dc}$ value is required for the particular operation. According to this measurement and monitoring, dc value coming from the source can be changed. That's why, this testing is done. Here, there are two DC input channels: DC1 and DC2. These two input channels are processed through the procedure given in Figure 15 below.

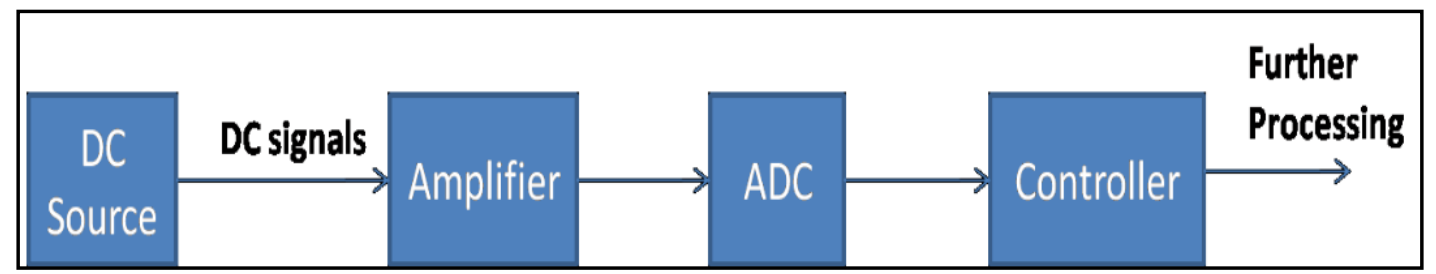

Fig. 15 Block Diagram representing processing of DC inputs 
This figure shows that a DC source is continuously providing DC signal to the AI section. These signals are then passed through buffer and digitized with the help of ADC. These digitized signals are then sent to the controller. These signals are continuously measured and monitored.

\subsubsection{DC1 and DC2 Measurement}

This testing is done to check whether the DC signal supplied by the DC source is measured correctly by the controller or not. The corresponding steps for DC voltage measurement are as follows:

Step 1: A number of DC input voltages are applied at the input of AI section from DC source.

Step2: The ADC count, corresponding to the applied input, is calculated by using the formula given in (1).

Step3: At the output of the ADC, the DC voltage is observed which should be equal or nearly equal to the applied input voltage.

Step4: The \%age of error is calculated between applied input DC voltage and the observed DC output voltage for each input case.

Step5: Graphs are plotted between Input Voltage and output voltage for both DC1 and DC2 channels as shown in Figure 16.
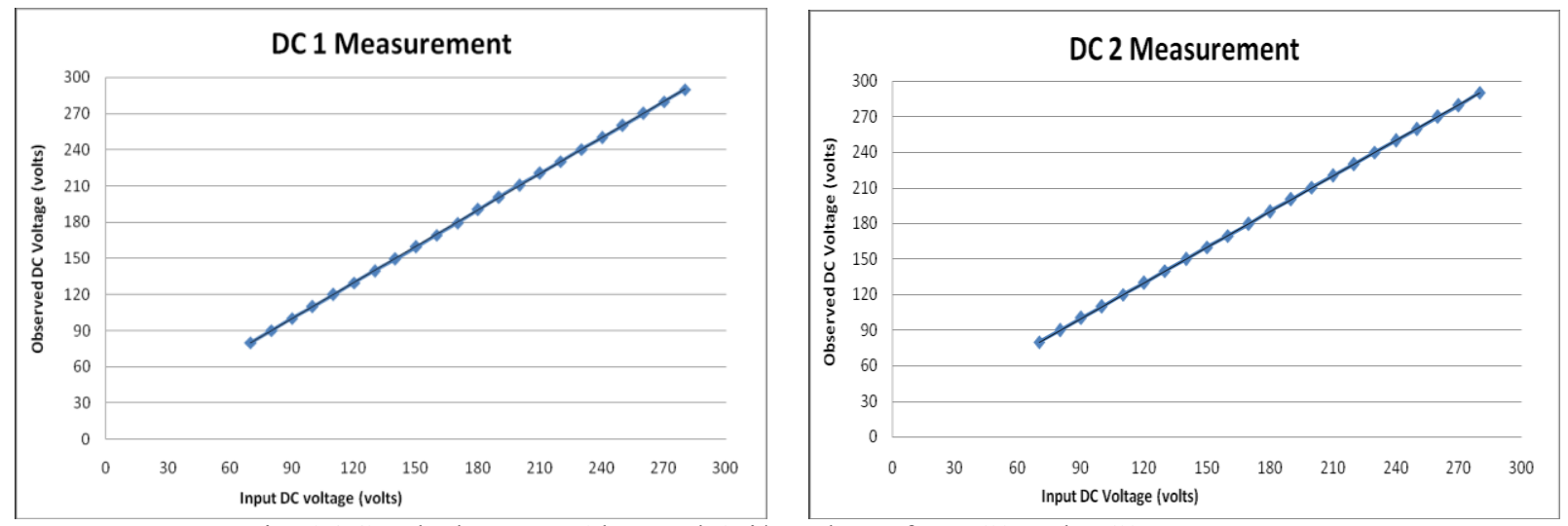

Fig. 16 Graphs between Observed \& i/p voltage for DC1 and DC2 measurement

These figures show that the graph between applied input voltage and the observed output voltage is linear. It means the observed DC output voltage follows the applied DC input voltage which is the desired result. Thus, the controller accurately measures the coming DC voltage input.

\section{Conclusion}

A stand alone RTU has been proposed whose each section analog, digital, communication and power supply are on a single PCB. This results in less wiring, low cost and small size. Analog section is the most critical one. In it, the inputs coming from the field have to be converted in a value that can be detected and measured by the controller accurately. The testing is done for auxiliary inputs, current inputs, voltage inputs and DC inputs. From these testing, following conclusions can be made. The controller measures auxiliary inputs (4-20mA) coming from the field accurately in terms of voltage or current accordingly for all the 3 input channels with minute difference that can be neglected. PT has been used for converting high voltage of $110 \mathrm{~V}$, coming from the field, into $0.2 \mathrm{~V}$ (threshold value that can be detected by the controller). PT testing showed that after setting the proper values of resistor network, $110 \mathrm{~V}$ can be accurately detected by the controller using ADC. Testing has also been done for both $1 \mathrm{~A}$ and $5 \mathrm{~A}$ current inputs (coming from the field) that have to be converted into at least $0.2 \mathrm{~V}$ using CTs. The results indicate that after selecting the correct values of burden resistor R1 for $1 \mathrm{~A}$ input and both $\mathrm{R} 1, \mathrm{R} 2$ resistors for $5 \mathrm{~A}$ input, $0.2 \mathrm{~V}$ was obtained accurately for both $1 \mathrm{~A}$ and $5 \mathrm{~A}$ using ADC. Also, DC voltage (80$290 \mathrm{~V})$ is continuously supplied to the system which the controller cannot directly measure. The testing of both the DC1 and DC2 channels showed that the controller accurately measures these inputs also using $\mathrm{ADC}$ with minute difference that can be neglected.

\section{Acknowledgement}

Authors are thankful to Dr. Suraj Pardeshi (CGL, Mumbai) and Er. B. S. Singla (SLDC, Patiala) for their kind cooperation, guidance and help in this work. 


\section{References}

[1] Sanath Alahakoon, Lilantha Samaranayake, Thilakasiri Vijayananda, Mats Leksell, Remote Monitoring And Distributed Real-Time Control Via Ethernet, Proc. of the 11th National Conference on Machines and Mechanisms, Indian Institute of Technology Delhi, New Delhi, December 18-19, 2003.

[2] Wikipedia Admin. Team, "Remote Terminal Unit", http//en.wikipedia.org/wiki/remote terminal unit, accessed on April, 2012.

[3] Hairulzawan Hashim, Zainal AlamHaron, A Study on Industrial Communication Networking: Ethernet Based Implementation, International Conference On Intelligent And Advanced Systems, KLConvetion Centre, Kuala Lumpur, 25 - 28 November 2007.

[4] CHEN Qi, DING Tianhuai, LI Cheng, WANG Peng, Low-power wireless remote terminal design based on GPRS/GSM, Journal of Tsinghua University, Science \& Technology, China, Feb., 2009, 49(2).

[5] Chen Peijiang, Jiang Xuehua, Design and Implementation of Remote Monitoring System Based on GSM, Pacific-Asia Workshop on Computational Intelligence and Industrial Application (PACIIA), Vol. 1, 678-681, Wuhan, 2008.

[6] Hong-Chan Chang, Li-Chien Huang, Cheng-Chuan Chen, Cheng-Chein Kuo, Design and implementation of remote terminal unit for feeder automation system with high performance microcontroller, 6th IEEE Conference on Industrial Electronics and Applications, 382-386, Beijing, 2011.

[7] Muhammad Aamir, Javier Poncela, Muhammad Aslam Uqaili, B. S. Chowdhry, Nishat Ahmad Khan, Optimal Design of Remote Terminal Unit (RTU) for Wireless SCADA System for Energy Management, Wireless Personal Communications, 69(3), 999-1012, April 2013.

[8] Motorola Inc., A 16 Digital Input 110 V Module for the MOSCAD-L RTU, 1999. 